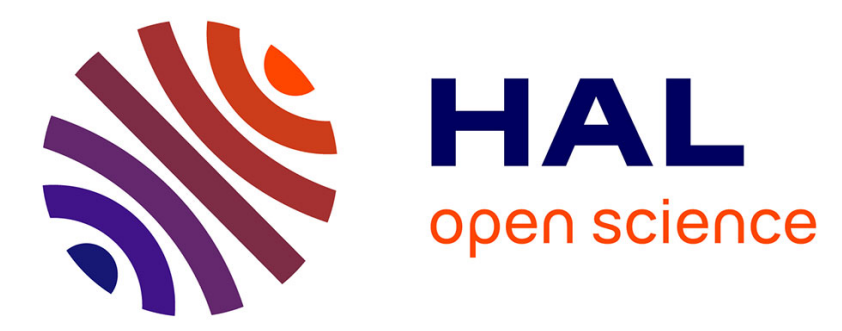

\title{
La définition des juridictions dans un espace terrestre et fluvio-maritime: Honfleur et ses environs (xie - xiiie siècles) \\ Laurence Jean-Marie
}

\section{- To cite this version:}

Laurence Jean-Marie. La définition des juridictions dans un espace terrestre et fluvio-maritime: Honfleur et ses environs (xie - xiiie siècles). Annales de Normandie, 2018, 68 (1), pp.7-26. 10.3917/annor.681.0007 . hal-02131717

\section{HAL Id: hal-02131717 \\ https://hal.science/hal-02131717}

Submitted on 16 May 2019

HAL is a multi-disciplinary open access archive for the deposit and dissemination of scientific research documents, whether they are published or not. The documents may come from teaching and research institutions in France or abroad, or from public or private research centers.
L'archive ouverte pluridisciplinaire HAL, est destinée au dépôt et à la diffusion de documents scientifiques de niveau recherche, publiés ou non, émanant des établissements d'enseignement et de recherche français ou étrangers, des laboratoires publics ou privés. 


\section{LA DÉFINITION DES JURIDICTIONS DANS UN ESPACE TERRESTRE ET FLUVIO-MARITIME : HONFLEUR ET SES ENVIRONS $\left(\mathrm{XI}^{\mathrm{E}}-\mathrm{XIII}^{\mathrm{E}}\right.$ SIÈCLES)}

Laurence Jean-Marie

Association Les Annales de Normandie | « Annales de Normandie »

2018/1 68e année | pages 7 à 26

ISSN 0003-4134

ISBN 9782902239399

Article disponible en ligne à l'adresse :

https://www.cairn.info/revue-annales-de-normandie-2018-1-page-7.htm

Distribution électronique Cairn.info pour Association Les Annales de Normandie.

(C) Association Les Annales de Normandie. Tous droits réservés pour tous pays.

La reproduction ou représentation de cet article, notamment par photocopie, n'est autorisée que dans les limites des conditions générales d'utilisation du site ou, le cas échéant, des conditions générales de la licence souscrite par votre établissement. Toute autre reproduction ou représentation, en tout ou partie, sous quelque forme et de quelque manière que ce soit, est interdite sauf accord préalable et écrit de l'éditeur, en dehors des cas prévus par la législation en vigueur en France. Il est précisé que son stockage dans une base de données est également interdit. 


\title{
La définition des juridictions dans un espace terrestre et fluvio- maritime : Honfleur et ses environs ( $\mathrm{xl} \mathrm{e}^{\mathrm{e}}-\mathrm{XIII}$ siècles)
}

\author{
LAURENCE JEAN-MARIE*
}

\begin{abstract}
I Honfleur a fait l'objet de travaux d'érudits, c'est marginalement que la période de l'émergence du port a été abordée compte tenu de la maigreur $\checkmark$ et de la disparité des sources. Leur mise en correspondance permet toutefois de proposer des éléments d'interprétation. Pour ce faire, il est nécessaire de replacer Honfleur dans un espace géographique plus large, celui de la rive gauche de l'embouchure de la Seine en aval de Quillebeuf. Les sources utilisées sont connues mais n'ont guère été confrontées concernant le partage des droits dans ce type d'espace où se rencontrent juridictions sur la terre ferme, l'estran et la zone fluviomaritime alors que les limites entre terre et eau varient.
\end{abstract}

L'accessibilité aux ports et l'escale dans ces derniers, entendus comme espaces permettant d'établir un lien entre mer et terre, est un réel problème dans l'estuaire de la Seine particulièrement mouvant où la navigation est chose malaisée. Des lettres patentes de Louis XI de 1480 indiquent que le port de Honfleur est « soumis à l'impetueulx flot et continuelle batterie de la mer » et qu'il est situé à l'embouchure « d'ung fleuve qui est ung des dangereux passaiges et destroiz du royaume ${ }^{\prime}{ }^{1}$. Les abords de la rive gauche sont décrits dans le routier de Pierre Garcie datant de l'extrême fin du XV ${ }^{\mathrm{e}}$ siècle. Il signale les bancs dont certains assèchent à marée basse (fig. 1). L'entrée en Seine ne peut se faire qu'avec la marée montante sauf pour un petit navire. La posée de Vasouy, à l'ouest de Honfleur, a une hauteur de fond de 4 brasses seulement à marée basse. Afin d'éviter les hauts-fonds, de permettre aux marins de se situer au mieux et de rester dans un chenal, les repères et les alignements ou les angles qu'ils forment entre eux et avec le navire sont précisément indiqués. Le passage vers le sud, du côté de Honfleur, est balisé par des pointes qui sont autant de repères : pointe de la Rocque de Risle, de Honfleur et celle qui « s'appelle Pissouse » (située à l'ouest de Honfleur) ${ }^{2}$.

* Normandie Univ, UNICAEN, CNRS, CRAHAM.

1 C. BrÉARd, Les archives de la ville de Honfleur, Paris, Picard, p. 48.

2 Pierre Garcie, dit Ferrande, Le grand routtier et pyllotage et encrage de la mer : tant ses parties de France, Bretaigne, Angleterre, Espaigne, Flandres, haultes Allemaignes, avecques les dangers des 
Dans cet espace mouvant, les modifications de la configuration des contacts entre mer et terre sont fréquentes. Un acte de 1256 fait état de potentielles évolutions concernant Crémanfleur et Fiquefleur, situés à proximité de Honfleur vers l'amont. Ces ports peuvent être naturellement améliorés par l'action de l'eau et de la mer sans le travail de l'homme ${ }^{3}$ mais l'eau peut également les obturer ${ }^{4}$. Les ports de la zone sont situés au débouché de cours d'eau côtiers; la Claire à Honfleur, l'Orange dans l'actuelle commune de la Rivière-Saint-Sauveur et la Morelle à Fiquefleur. Des problèmes d'atterrissement par transport de galets ou de sable colmatant un chenal peuvent gêner voire interdire l'accès aux ports ; inversement un transport rapide est susceptible d'en dégager les accès. Les modifications touchent évidemment Honfleur et des documents bien postérieurs le montrent. Des lettres de Charles VIII du 4 janvier $1497^{5}$ évoquent que «depuis demy an ença la riviere de Seyne passant par devant ladite ville de Honnefleu a changé son cours et lieu par lequel elle avoit touisours accoustué de passer et courir et vient a présent passer et courir joignant les dites gectées ». Les évolutions, favorables ou défavorables aux activités humaines, témoignent bien d'une situation instable ${ }^{6}$.

Malgré ces difficultés, la situation de Honfleur et des petits ports voisins revêt un intérêt évident compte tenu de l'importance de l'axe de la Seine et du potentiel économique qu'offrent les zones fluviomaritimes. C'est souvent en creux que les sources le montrent. Elles mettent en évidence la variété des espaces sur lesquels les limites de l'exercice des juridictions doivent être déterminées. La précision des indications témoigne de la diversité des droits, possession de la terre et droits d'usage, et de la nécessité d'en déterminer très exactement l'étendue compte tenu de la variabilité des confins entre terre et eau. Les conflits entre les autorités seigneuriales et leur résolution rendent compte du potentiel économique de la zone et nécessitent redéfinitions des droits et de leurs limites géographiques. De cet ensemble, émergent peu à peu les ports qui sont ou deviennent des enjeux de pouvoirs.

ports, havres, rivières, chenal des parties \& régions susdites, Reproduction de l'édition de Poitiers, sire Enguilbert de Marnef, 1521, BnF, NUMM- 54143, fol. 82r.

3 [...]dictos portus cum pertinentiis suis in statu in quo modo sunt et erunt, si per aquam et mare sine manus opere poterunt emendari (L. Delisle, Cartulaire normand de Philippe-Auguste, Louis VIII, Saint-Louis et Philippe le Hardi, Caen, Hardel, Mémoires de la Société des antiquaires de Normandie, XVI, 1872, n 545, note, p. 100 ; « Acte 3529 », dans SCRIPTA. Base des actes normands médiévaux, dir. Pierre Bauduin, Caen, CRAHAM-MRSH, 2010-2016. [En ligne] https://www.unicaen.fr/scripta/ acte/3529; L. Delisle, L. Passy, Mémoires et notes de M. Auguste Le Prévost pour servir à l'histoire du département de l'Eure, Évreux, Hérissey, 1862-1869, t. 2, p. 107 ; « Acte 5636 », dans SCRIPTA.

4 Si vero acciderit quod aqua dictos portus obturaverit, etc. (Ibid.).

5 C. BrÉARD, Les archives de la ville de Honfleur, op. cit., p. 51.

6 J. Mouchard, « De la voie navigable aux sites portuaires en basse vallée de Seine : maîtrise et gestion des accès (Antiquité - époque moderne) », dans E. Lalou, B. LePeuple et J.-L. Roch (dir.), Des châteaux et des sources: Archéologie et histoire dans la Normandie médiévale, Mélanges en l'honneur d'Anne-Marie Flambard Héricher, Publications des universités de Rouen et du Havre, 2008, p. 103-127. 


\section{LA RIVE GAUCHE DE L'ESTUAIRE DE LA SEINE : UN ESPACE PARTAGÉ}

C'est à partir des actes concernant le temporel de l'abbaye de Grestain, établie à quelques kilomètres en amont de Honfleur, que l'on peut tenter une première géographie des pouvoirs sur la rive gauche de l'estuaire de la Seine. Dans la pancarte de confirmation des possessions de l'abbaye par Guillaume le Conquérant en 1082, des biens et droits y sont situés. On en retiendra les plus importants et ceux qui ont un lien avec les activités maritimes ${ }^{7}$. Du don d'Herluin de Conteville, fondateur de l'abbaye, figurent des terres, bordiers et pêcheurs à Grestain ${ }^{8}$. Par donation de Robert, comte de Mortain (fils du premier et frère utérin du duc), la moitié de la coutume sur la Seine de Quillebeuf à Pillola Rocha (Saint-Samson-de-la-Roque); la moitié du marché de Fiquefleur, la moitié des salines de ce même lieu ; tous ses bourgeois et bordiers à Jobles, Fiquefleur, Crémanfleur et Honfleur ; la dîme des foires entre Risle et Touques; enfin, ce qu'il détient dans un moulin à Fiquefleur 9 . Des biens non identifiés issus de la dot de Fredesende, femme d'Herluin, sont donnés à l'abbaye au Neubourg ${ }^{10}$, à Cantelou et à Honnaville, soit à proximité de Honfleur ${ }^{11}$. Des bordiers à Fiquefleur et Crémanfleur et 20 areas pour faire le sel constituent un don d'Odon de Bayeux.

En 1189, Richard Cœur de Lion confirme les biens de l'abbaye. La charte précise les choses et fait état de possessions non explicitement mentionnées en 1082 dont certaines peuvent être rattachées aux donations originelles ${ }^{12}:$ du don de Guillaume le Conquérant, la chapelle Saint-Nicolas près de Honfleur ; de celui d'Herluin, 30 acres de terre à Grestain, des bordiers et pêcheurs et une forêt $t^{13}$. Suit encore une longue énumération de donations de Robert de Mortain. Elle comprend toutes les coutumes qu'il tenait en dominium sur la rive de la Seine depuis le gord ${ }^{14}$ de Quillebeuf jusqu'à Noirport, c'est-à-

7 D. Bates, V. Gazeau, « L'abbaye de Grestain et la famille d'Herluin de Conteville », Annales de Normandie, 1990, $\mathrm{n}^{\circ}$ 1, p. 5-30 ; D. BATEs, Regesta regum anglo-normannorum. The acta of William I, 1066-1087, Oxford, Clarendon Press, 1998, n 158, p. 517-524.

8 En outre, une forêt proche du monastère et une partie d'une autre sur le Mont-Saint-George (Fiquefleur) ; à Foulbec un bordier et une petite forêt, à Conteville une acre de pré, à Berville un marais appelé Apulia.

9 S'y ajoutent des biens des biens à Grestain et Carbec (commune de Fatouville-Grestain).

10 Il s'agit probablement de de la « Rivière du Neufbourg » correspondant au hameau de la Rivière (commune de La-Rivière-Saint-Sauveur) située dans la paroisse de Saint-Léonard de Honfleur dans un acte de 1634 (C. BrÉARD, L'abbaye Notre-Dame de Grestain, Rouen, A. Lestringant, 1904, PJ XC).

11 Les deux dans la commune actuelle de Gonneville-sur-Honfleur.

12 On reprend ici les donations essentielles au propos.

13 De plus une terre à Grestain et la moitié du moulin de Carbec.

14 On appelle gord un réservoir où l'on conserve du poisson (C. BRÉARD, L'abbaye de Notre-Dame de Grestain..., op. cit., p. 26). On rencontre le terme en Bretagne aussi et, sur le reste de la côte atlantique, il porte d'autres noms (M. Bochaca, B. Arízaga Bolumburu et A. Gallicé, « Les pêches maritimes dans le golfe de Gascogne... », op. cit.). Il s'agit probablement ici d'un espace de taille respectable puisqu'il sert de repérage topographique ; le poisson y est probablement conservé vivant comme le suggère l'association suivante entre pêcherie et gord: Dedi quoque piscariam, quod vulgo gordum dicitur, apud Oscellum villam (Recueil des actes des ducs de Normandie de 911 à 1066, M. Fauroux (éd.), Caen, 
dire toute la coutume de l'eau salée, et, une fois par semaine, le flot montant et descendant avec tout ce qui sera trouvé dans les filets quelles que soient la terre ou la domination de ceux qui auront envoyé leurs filets dans l'eau (donc un prélèvement hebdomadaire sur toute la pêche effectuée dans cette zone par tous les pêcheurs) ${ }^{15}$; tous les esturgeons péchés dans « toute son eau " sauf le premier de l'année qui revient au duc; le premier saumon, la première alose et la première lamproie pris dans les filets et la moitié d'un marsouin capturé avant la Quadragésime ; tout le varech - le droit de s'approprier épaves et cargaisons rejetées par la mer - entre l'Épine de Berville ${ }^{16}$ et Honfleur « où que la mer le jette, jusqu'à une certaine terre au-delà du galet et, s'il parvenait en deçà de cette tenure, là où il n'y a pas de propriété des moines, il serait soumis à amende et justice ». Avec le bénéfice du varech, figure le droit de porter dans l'église de l'abbaye le corps et l'argent de tout homme mort dans un navire ancré dans cet espace ${ }^{17}$. L'énumération se poursuit avec la terre et le galet recouvert habituellement par le flot coutumier avec toute la coutume avant une certaine terre ${ }^{18}$; sur la terre " sèche » (terra sicca), ce que Robert possède sur la rive, à savoir des bordiers et bourgeois à Jobles, Crémanfleur, Fiquefleur et Honfleur; tout le tonlieu depuis l'Épine de Berville jusqu'au port de Honfleur payé par les étrangers ${ }^{19}$; le marché de Fiquefleur et la foire de cette villa avec toute la coutume; la dîme des foires entre Risle et Touques et le tiers du moulin de Fiquefleur ${ }^{20}$. Les areas ${ }^{21}$, le galet et les coutumes appartenant à ces areas sont exempts de taxe depuis l'Épine de Berville jusqu'à l'eau

Caron, Mémoires de la Société des antiquaires de Normandie, t. XXXVI, 1961, n 85 ; « Acte 1528 », dans SCRIPTA).

15 Ex dono Roberti comitis Moretis filii Herlewini, fratris Willelmi, regis Anglorum et ducis Normannorum, omnes consuetudines et custumas quas tenebat de dominio suo et fratre Willelmo rege in riparia Secane a gardo de Kilebuef usque ad Nigrum portum id est totam custumam aque saline, semel in septimana, ad Unumfletum, ascendentem et recedentem quicquid in retiis inventum fuerit de cujuscumque terra aut dominatu fuerint omnes qui retia in aquam miserint (C. BRÉARD, L'abbaye de Notre-Dame de Grestain, op. cit., PJ I, 1189, confirmation de donations de Robert comte de Mortain, demi-frère de Guillaume le Conquérant). On propose de corriger la transcription de Charles Bréard qui lit Unumfletum et considère qu'il s'agit de Honfleur, par unum fletum, un flot, ce qui rend compréhensible la précision ascendentem et recedentem. Cela correspond en outre à la graphie de la copie moderne de la BnF (ms lat. 12778, fol. 244v).

16 L'Épine de Berville paraît désigner l'avancée de terre sur la rive gauche de la Risle, au niveau de sa confluence avec la Seine, qui contraint la rivière à effectuer un méandre.

17 [...] ]et si mortuus fuerit aliquis in nave tenente per anchoram corpus ejus cum pecunia ad ecclesiam monachorum deportetur (C. BRÉARD, L'abbaye de Notre-Dame de Grestain, op. cit., P.J. I, 1189, acte de Richard Cœur de Lion, confirmation de donations de Robert comte de Mortain, demi-frère de Guillaume le Conquérant).

18 Dedit quoque terram et galeum quantum fluctus consuetudinarie ascendit cum omni custuma cujuscumque antea certana terra fuerit sicut dominus suus rex Willelmus ei donavit (C. BRÉARD, L'abbaye de Notre-Dame de Grestain, op. cit., P.J. I, 1189). Il est précisé, pour ce dernier point que c'est le duc Guillaume qui céda auparavant ces droits à son demi-frère à Herluin de Conteville.

19 [...] et totum theloneum quod fiet a spina Berville usque ad portum de Hunefluctu sub via et in via de omnibus alienis.

20 Et le moulin de Grestain ; la suite ne concerne pas le secteur.

21 C. Bréard commet une erreur en écrivant acras. 
de Honfleur ${ }^{22}$. Le tonlieu sur le sel doit revenir au duc lorsqu'il est transporté par terre et aux moines lorsqu'il l'est par mer $^{23}$. Il appartient à ces derniers à proximité de la fontaine de Foulbec jusqu'à la mer et jusqu'au pont (sur la Risle probablement) sauf celui du pain, du sel et du poisson frais. Il en est de même de la moitié du tonlieu du port de Honfleur concernant ce qui vient par mer ou repart. Figurent encore des biens au Neubourg, à Cantelou et à Honnaville et 15 acres de pré à Conteville.

Le contenu de la confirmation de 1189 est bien plus détaillé que celui de la pancarte de de 1082. L'abbaye n'est pas détentrice d'un ensemble cohérent de terres agricoles dans ce secteur ${ }^{24}$. En revanche, elle dispose de très nombreux biens et droits et il est manifeste que ceux liés aux activités maritimes sont particulièrement étendus. Ils apparaissent plus nettement dans l'acte de 1189, probable signe de l'essor de ces dernières et de la nécessité de définir les prérogatives de chacun dans des espaces précis, même s'il faut aussi prendre en compte le fait que les donations à l'abbaye ont pu être complétées postérieurement à 1082 .

Certains droits débutent à Quillebeuf, en amont, là où le resserrement des rives marque la fin de l'estuaire de la Seine (fig. 1). Dans le premier texte, le bénéfice de la moitié de la coutume sur la Seine s'étend en aval jusqu'à SaintSamson-de-la-Roque et, dans le second, c'est l'ensemble des coutumes que possède l'abbaye dans une zone plus vaste vers l'aval, allant jusqu'à Noirport. Le droit de varech s'étend sur une zone moins étendue en amont, qui commence à l'Épine de Berville et s'étend jusqu'à Honfleur, en aval. À Honfleur, seul lieu qualifié à deux reprises de port dans l'acte 1189, l'abbaye a quelques possessions et Honfleur sert aussi de limite aux droits qu'elle exerce. Pour le droit de varech, il est explicitement précisé qu'il s'applique jusqu'à une certaine terre, qualifiée de tenure, avec menace de poursuites judiciaires si l'abbaye ne respecte pas cette règle. Le tonlieu n'est perçu par l'abbaye qu'à partir de l'Épine de Berville, en amont, mais jusqu'au port de Honfleur, non compris, puisqu'à cet endroit elle n'en perçoit que la moitié. Le souci de précision des limites de juridiction qui ressort de l'acte de 1189 peut être le témoignage en creux de conflits attestés dans les sources plus tardives et dont Honfleur est un point nodal. Ces définitions spatiales posent la question de la position relative de Noirport, toponyme aujourd'hui disparu, et de Honfleur, sur laquelle il faudra revenir.

L'abbaye dispose, d'une part, de biens (salines, terres, bois, moulins, etc) et, d'autre part, sur des zones plus étendues, de droits et coutumes. L'ensemble dessine des espaces différenciés : le fleuve lui-même, l'estran, les ports et la terre

22 La formulation fait douter du contenu de la donation mais il semble que ce soit bien l'exemption de droits qui est attribuée plutôt que la donation des espaces eux-mêmes.

23 Il manque plusieurs mots à l'item suivant d'après C. Bréard.

24 D. Bates, V. Gazeau, «L'abbaye de Grestain et la famille d'Herluin de Conteville», op. cit. 
ferme (terra sicca). Les droits de l'abbaye n'ont pas les mêmes limites selon qu'il s'agit de l'estran ou du fleuve sur le cours duquel l'abbaye exerce une juridiction comme le confirme un acte de 1287 dans lequel l'abbé de Grestain évoque « nostre eaue de Sainne » entre l'Épine de Berville et Noirport ${ }^{25}$. L'estran, qui comprend le galet et auquel on peut associer les areas où sont installées les salines, est une zone intermédiaire, en partie appropriée et dans tous les cas soumise à des droits d'usage sur la pêche ou peut-être la pâture ${ }^{26}$. Elle connaît des variations importantes en dehors même du rythme quotidien. Un exemple l'illustre. En amont de l'Épine de Berville, les droits sont partagés entre les abbayes de Jumièges et de Grestain. Dans un accord de 1259 entre les deux monastères pour la répartition des droits entre l'Épine de Berville et la fosse de Foulebec, soit dans la zone de confluence entre Risle et Seine, il est prévu que si l'eau se retirait de manière à ce que l'on puisse labourer la terre, cette dernière serait aux mains de Jumièges ${ }^{27}$.

Au bilan, on peut proposer l'hypothèse que la délimitation précisément bornée de l'exercice des droits sur le fleuve et l'estran porte la trace d'un ancien espace juridique aux mains des ducs, progressivement démembré, comprenant une série de petits ports d'échouage, mal différenciés encore à la fin du XI ${ }^{\mathrm{e}}$ siècle et pour lequel les droits sur le commerce étaient rassemblés en un unique tonlieu levé tout au long de la zone. Les radicaux communs aux toponymes Honfleur, Fiquefleur et Crémanfleur pourraient être un indice de cet ensemble cohérent datant d'une même époque ${ }^{28}$. On peut penser qu'existaient sur ce bout de côte des droits ducaux, passés à Herluin de Conteville puis, en partie, à l'abbaye de Grestain au XI ${ }^{\mathrm{e}}$ siècle. Sur la rive droite, il est frappant de constater que la seigneurie des Tancarville s'exerce jusqu'au « figuier Daurechier » au $\mathrm{Xv}^{\mathrm{e}}$ siècle au moins ${ }^{29}$. Or, Lorcher est situé au droit de Honfleur sur la rive nord ${ }^{30}$. Que cet ensemble cohérent ait existé ou non, il est évident qu'à la fin du $\mathrm{XI}^{\mathrm{e}}$ siècle, il n'est plus et a laissé place à un partage des juridictions.

25 C. BRÉARD, L'abbaye de Notre-Dame de Grestain, op. cit., PJ XVIII ; cette juridiction sur le cours du fleuve est encore confirmée en 1411 lorsque l'abbé reconnaît devoir chaque année un esturgeon au roi « pour cause de la moitié de la rivière de Sainne, au costé depuis le gord de Quillebeuf jusques à un lieu nommé le Noir-Port » (Ibid., PJ XXXV, 1411 et XL, 1424).

26 Un acte de 1615 évoque les pâturages existant sur les bancs de Seine (Ibid., PJ LXXXV).

27 «Et ce d'aventure il advenoit que par aucun temps l'eau se retiroit en aucun lieu en telle manière que l'on y peult labourer, ladite terre demeureroit a iceulx abbé et couvent de Jumièges » (Ibid., PJ XV, 1259). L'emploi du français fait toutefois douter de la date.

28 Les radicaux de ces toponymes peuvent être d'origine scandinave.

29 C. Bréard, L'abbaye de Notre-Dame de Grestain, op. cit., PJ XXXIV, 1450 et E. Deville, Histoire du château et des sires de Tancarville, Rouen, Periaux, 1834, p. 151.

30 Actuelle commune de Gonfreville-l'Orcher. 


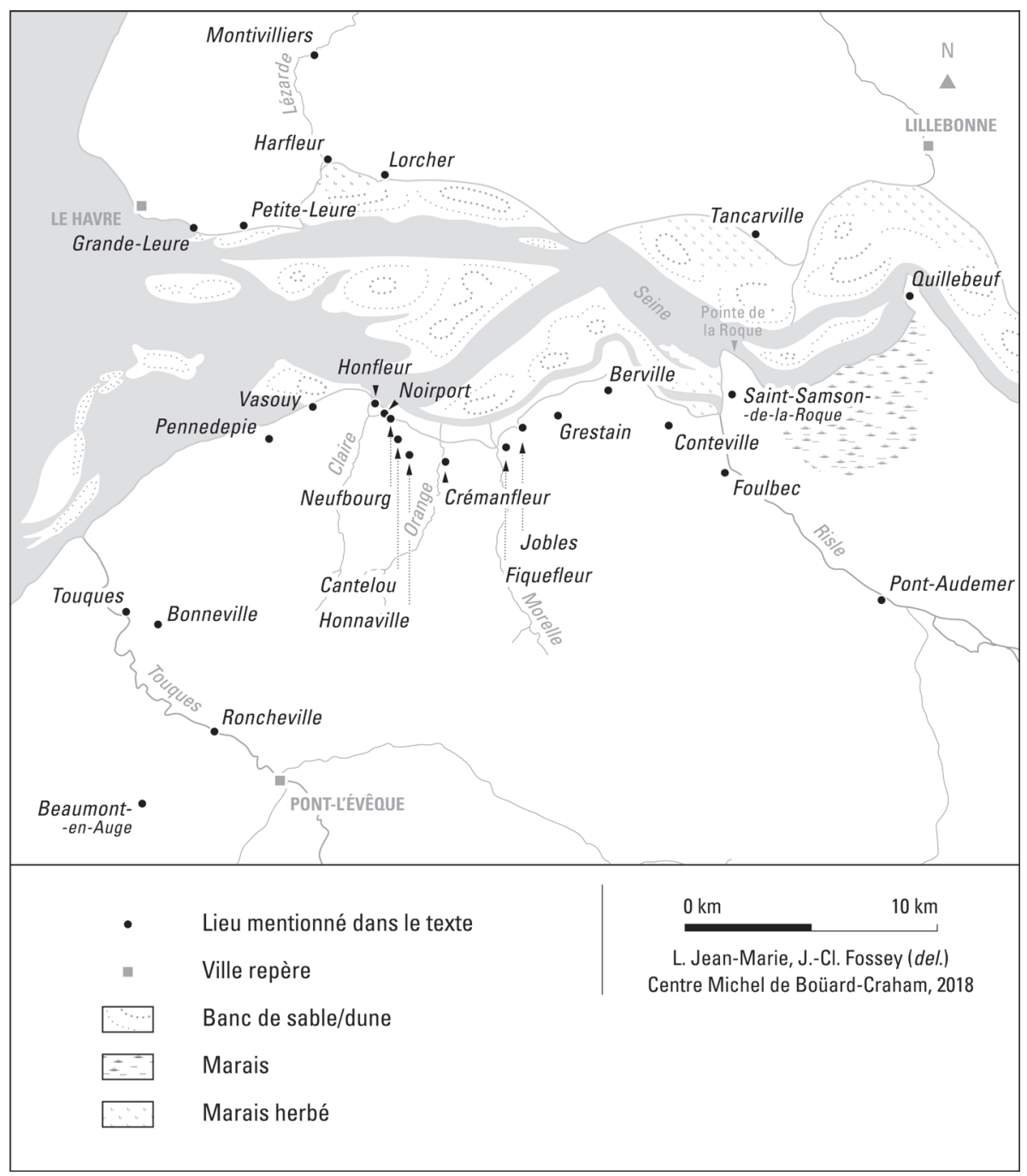

Fig. 1 - L'embouchure de la Seine : essai de restitution de la topographie ${ }^{31}$

\section{UNE DÉTERMINATION PRÉCISE DES LIMITES DE JURIDICTION}

Des informations complémentaires sont fournies dans des actes postérieurs confirmant que les limites de juridiction font l'objet de discussions. C'est notamment avec les Bertran, qualifiés de seigneurs de Honfleur au XIII ${ }^{\mathrm{e}}$ siècle,

31 La restitution des espaces a été réalisée à partir des cartes modernes et spécialement de celle de Georges Boissaye du Bocage (Carte de l'embouchure de la rivière de Seine, 1697, BnF, département Cartes et plans, GESH18PF37DIV3P4). 
que l'abbaye de Grestain a maille à partir. Dans un accord passé en 1287 entre Robert Bertran et l'abbaye, figure une stricte détermination du droit de varech de chacune des parties: Grestain en bénéficie depuis l'Épine de Berville jusqu'à la maison détenue autrefois par Robert Hachart à Honfleur, en aval ${ }^{32}$. Cet emplacement correspond très certainement à la limite de l'acte de 1189 qui mentionnait « une certaine terre au-delà du galet ». En accord entre les parties en 1287, a été placée à cet endroit une bonde (borne $\left.{ }^{33}\right)$, témoignage de la précision de la limite ${ }^{34}$. Le varech entre cette dernière et Noirport, donc situé en aval, revient à Robert Bertran. La maison où est placée la borne est identifiée comme étant à Honfleur, en amont de Noirport qui serait alors considéré comme intégré à la ville, potentiel signe du développement topographique de cette dernière dont témoigne aussi le fait qu'une terre ait été remplacée par une maison. En 1325, c'est le jardin de Raoul Le Mire à Honfleur qui sert de limite de juridiction entre Honfleur et, à l'amont, le monastère Sainte-Marie de Quillebeuf ${ }^{35}$. À la suite de Jean Mallon, on peut proposer l'hypothèse que ce jardin est situé à Noirport. Là encore, le texte détermine très précisément la limite.

Lacte de 1287 précise en outre que coutumes, franchises et seigneuries appartiennent à Robert Bertran entre la Croix du Perir et Noirport. Dans ce même espace, Grestain dispose toutefois des coutumes des marchandises "faictes à sèche terre [...] hors de la ou flo monte et retraict ", selon la donation qu'en a faite Robert de Mortain ${ }^{36}$, du droit de varech. On peut à bon droit en déduire que les Bertran y détiennent les revenus des taxes sur le commerce maritime. La croix du Périer dite Nogueron constitue par ailleurs la limite orientale de la prévôté de Honfleur d'après le tarif des droits de coutume, connu par une version de 1527 mais dont le contenu est en partie antérieur ${ }^{37}$. Son emplacement est inconnu ${ }^{38}$. Un accord du milieu du $\mathrm{Xv}^{\mathrm{e}}$ siècle entre le comte Tancarville et le seigneur de Roncheville (et de Honfleur) ${ }^{39}$ indique

32 C. BrÉArd, L'abbaye de Notre-Dame de Grestain, op. cit., PJ XVIII ; voir aussi C.-M. de Robillard de Beaurepaire, De la Vicomté de l'eau de Rouen et de ses coutumes aux XIII et XIV siècles, Évreux, Hérissey, 1856, p. 177-178.

33 Dictionnaire de Godefroy (complément), bodne. Anglo-Norman Dictionary, bonde.

34 Du côté aval, les délimitations font aussi l'objet de contestations et de transactions comme celle entre Guillaume de Tancarville et Grestain en 1409 faisant allusion à de précédents procès en 1310 et 1390. Les droits de Grestain y sont définis comme s'étendant entre la Croix de la Devise, précisant la limite du côté de Quillebeuf, et Noirport (C. BRÉARD, L'abbaye de Notre-Dame de Grestain, op. cit., PJ XXXIV). La croix est située entre le Marais-Vernier et Saint-Aubin-sur-Quillebeuf.

35 J. Mallon, Un port normand au Moyen Âge. Honfleur du XIII siècle à la fin du XV siècle, thèse de l’École des chartes, 1926, p. 65 ; Arch. nat., JJ 62, fol. 223v, nº 405.

36 On peut établir un parallèle avec la mention de la terra sicca dans l'acte de 1189 qui sert à définir les droits de l'abbaye sur la terre ferme depuis Berville jusqu'à Honfleur

37 C. BrÉard, L'abbaye de Notre-Dame de Grestain, op. cit., p. 123-124 ; J. Mallon, Un port normand au Moyen Âge..., op. cit., note p. 65-66.

38 E. Poret de Blosseville, Dictionnaire topographique du département de l'Eure, Paris, 1877.

39 À cette date, ce sont les Rocheguyon, héritiers par les femmes des Bertran (N. PALlu, Honfleur et son arrière-pays, chronique d'un espace militaire (1367-1530), Université Paris-Diderot Paris 7, M. Arnoux (dir.), 2012, vol. 1, p. 224). 
toutefois que ce dernier a droit de prendre les coutumes à terre de Honfleur à la Ruelle où était la Croix Nagueron ${ }^{40}$. Dans son Histoire de la ville de Honfleur, Pierre Thomas écrit, sans citer sa source, que la croix du Périer était située sur le bord de la Morelle ou rivière de Fiquefleur, endroit où elle se jetait à ce moment ${ }^{41}$. Un toponyme «la Croix de Pierre » existe à Fiquefleur sur le cadastre napoléonien mais il est difficile d'affirmer qu'il y ait un lien ; un ruisseau à Fiquefleur, se jetant dans la Morelle, porte le nom de la FontainePerrier.

L'enchevêtrement des droits est évident et la disparition de certains toponymes et microtoponymes complique la compréhension. Des mentions postérieures permettent de préciser la situation de Noirport, les hypothèses émises pour le placer immédiatement à l'est de Honfleur sont pertinentes, mais la détermination du site exact mérite discussion ${ }^{42}$. En 1424, l'abbé de Grestain reconnaît devoir au roi le premier esturgeon pêché dans la Seine "depuis le gord de Quillebeuf jusqu'à un lieu nommé le Noir-Port à nous appartenant ", confirmant que Noirport n'est pas seulement une limite mais est aux mains de l'abbaye ${ }^{43}$. Surtout un dénombrement des fiefs de l'abbaye de 1646 évoque des droits depuis le "gord de Quillebeuf, jusques au Noir-Port qui seroit à présent la grosse tour de Honnefleur ${ }^{44}$. Dans un bail de l'abbaye pour des droits de pêche sur la Seine en 1607, la zone est délimitée « depuis la Tour Carrée dudict Honnefleur jusqu'au Noirport près Quillebeuf ". Noirport n'est plus ici connu et le texte est erroné quand il le situe près de Quillebeuf. Reste qu'à deux reprises, le lieu est assimilé à une tour. La Tour carrée de Honfleur a été construite au XIV $v^{\mathrm{e}}$ siècle lors des aménagements du port réalisés par Charles V. Elle fait partie du système de fortification qui entoure l'Enclos et constitue la limite orientale du port à ce moment (fig. 2 et 3$)^{45}$. De façon évidente, le toponyme Noirport a disparu à la fin du Moyen Âge, sans doute en raison des aménagements du port de Honfleur et des travaux de fortifications. Il ne figure sur aucune carte conservée. Il est alors nécessaire d'identifier le lieu à partir d'autres éléments de la topographie. L'exploitation des sources du XIve siècle permettrait peut-être de donner des éléments de compréhension de ce qui semble aussi impliquer une redéfinition des limites de juridiction.

40 C.-M. de Robillard de Beaurepaire, De la Vicomté de l'eau de Rouen, op. cit., p. 178-179.

41 P. Thomas, Histoire de la ville de Honfleur, Honfleur, Dupray, 1840, p. 64 ; son cours aujourd'hui est celui du chenal antérieur le long de la Seine.

42 C. BRÉARD, L'abbaye de Notre-Dame de Grestain, op. cit., note 7, p. 123-124 ; J. Mallon, Un port normand au Moyen Âge..., op. cit., p. 66 ; ce dernier identifie Noirport au Havre Neuf aménagé postérieurement à l'est de l'Enclos.

43 C. BRÉARD, L'abbaye de Notre-Dame de Grestain, op. cit., PJ XL.

44 Ibid., PJ XCIV ; la baronnie de Grestain y est définie comme s'étendant dans les paroisses de «Carbec, Grestain [...] Conteville, Ablon, Ableville, Esquainville, Cremanville, Honnaville, la Rivière du Neuf-Bourg, parties des faulbourgs de Honnefleur et ès partie circonvoisines ».

45 N. PALlu, Honfleur et son arrière-pays, op. cit., vol. 1, p. 34. 


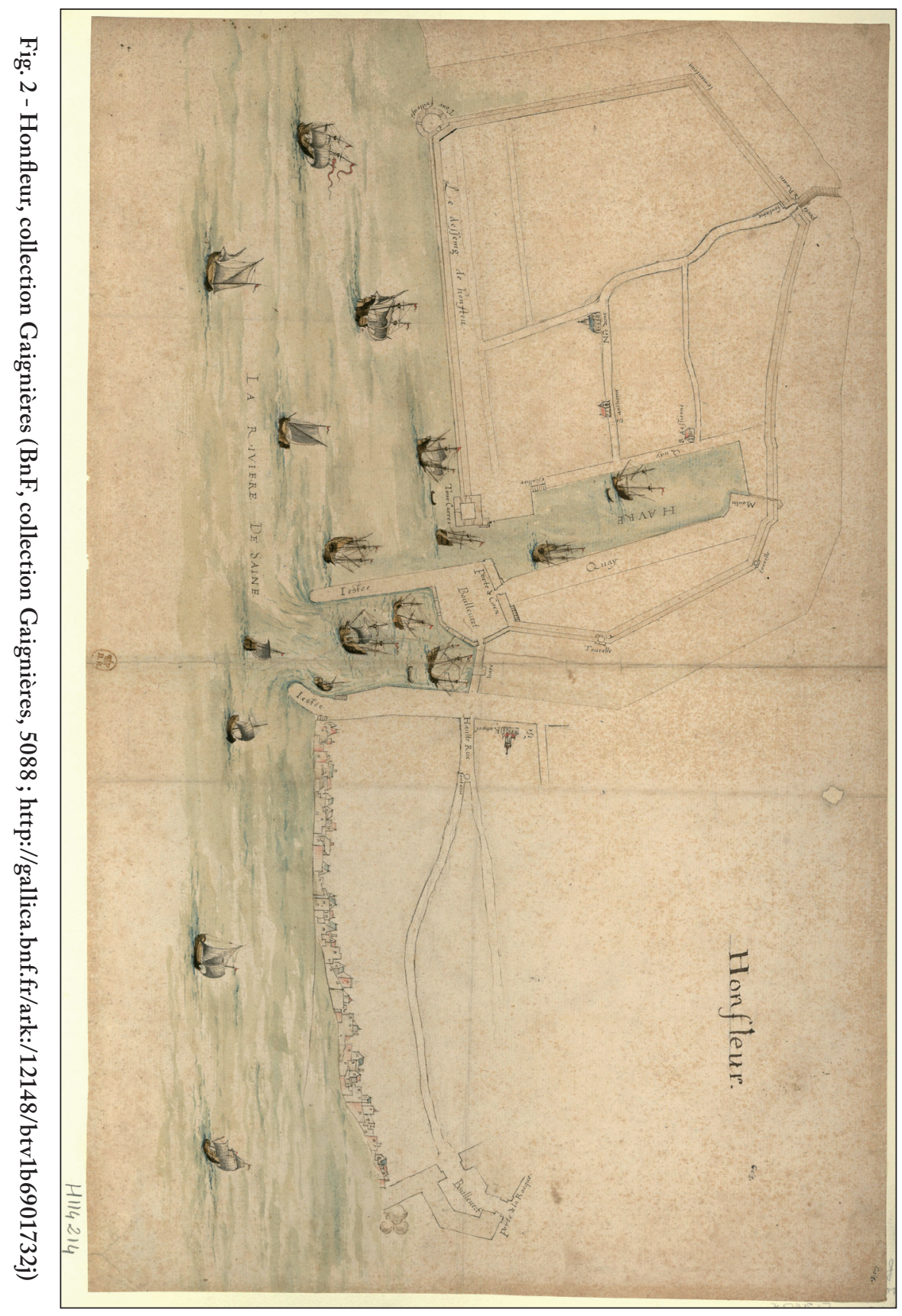




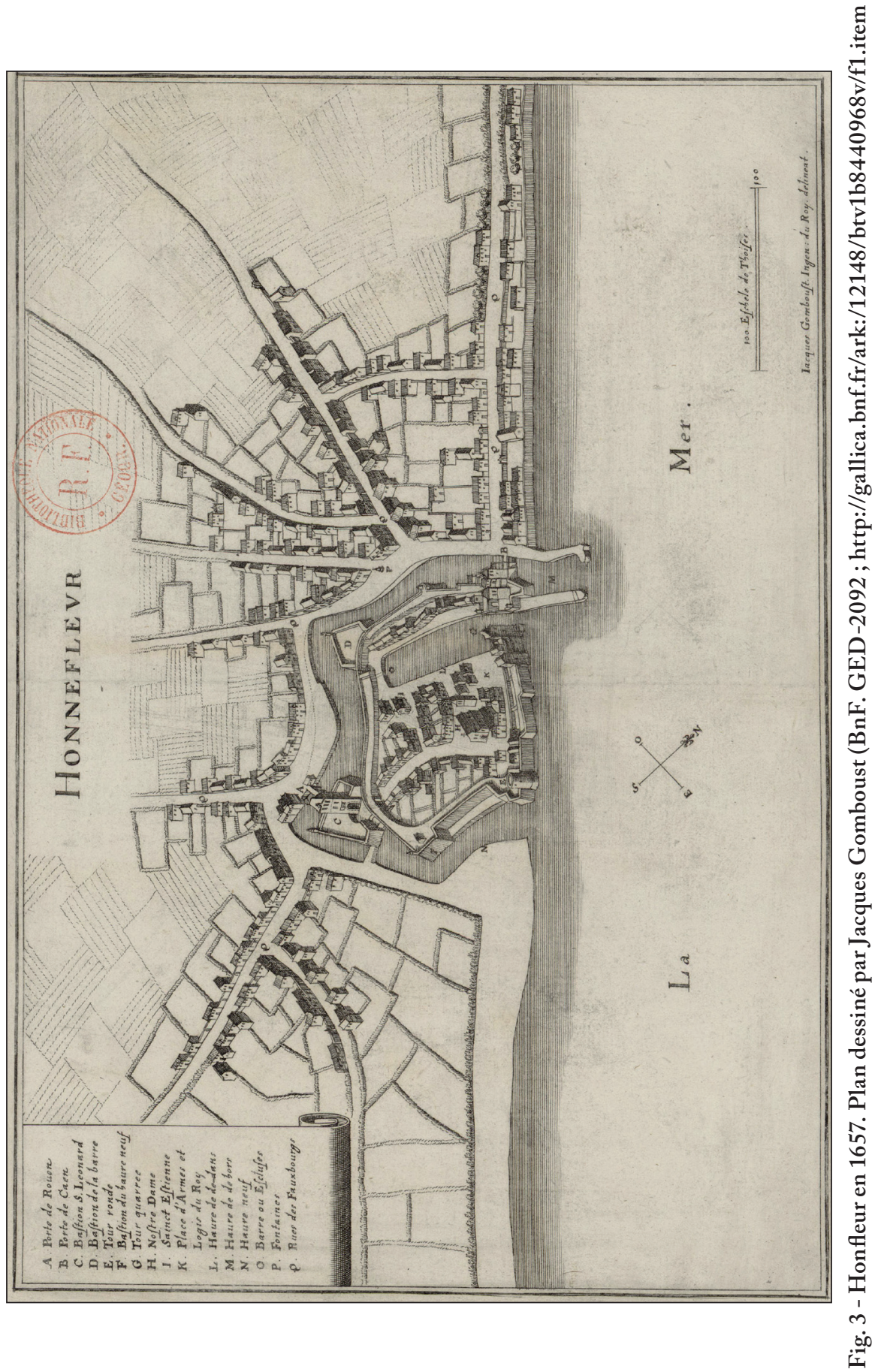


Tout cela pose la question de la topographie du port de Honfleur avant les aménagements de la seconde moitié du XIV siècle. Rares sont les sources qui fournissent des éléments. Dans un acte sans date, Robert Bertran (de Barneville) cède à Jean Le Tort ${ }^{46}$ de Honfleur, pour le prix de 50 sous tournois, une terre située devant son cellier de la villa de Honfleur, à côté du pont et de la terre où se trouvent les marches du cellier. Le bénéficiaire devra y édifier une maison et sera redevable annuellement de deux deniers monnaie usuelle, somme modique témoignant de la volonté de développer l'habitat. En 1229, des rentes sur deux maisons situées à côté du port sont données à l'abbaye de Grestain par Robert le Vavasseur de Vasouy afin de sauver son corps des dangers de la mer ${ }^{47}$. Avant 1234, Jean Ansere et sa femme vendent à Thostan de Leure deux masures avec places dans la paroisse Saint-Étienne, donnant sur le chemin devant et, derrière, sur le havre (habulum), de même que deux sous de rente à percevoir sur une maison située à côté des masures ${ }^{48}$.

Le port est installé au débouché de la Claire que le pont cité enjambe probablement, mais le cours d'eau a sans doute plusieurs bras. Le port pourrait alors être situé à l'emplacement du «Havre de dehors » du plan de Gomboust ${ }^{49}$. Il est possible qu'au XIII ${ }^{\mathrm{e}}$ siècle encore, existe un port sur un autre bras de la Claire correspondant peu ou prou au « Havre du dedans » sur le bord duquel est ensuite construite la Tour carrée, ce serait Noirport. La reconstitution de la géographie paroissiale permettrait peut-être de préciser les choses. Il n'est en effet pas établi que l'église Saint-Etienne soit située là où elle se trouve à la fin du Moyen Âge, à savoir dans l'Enclos. Son ressort et l'église elle-même pourraient être situés sur la rive gauche de la Claire avant la fondation de la paroisse Sainte-Catherine ${ }^{50}$. Les aménagements postérieurs, l'absence de données précises et les évolutions naturelles de la zone rendent toute reconstitution très hypothétique. Honfleur est un port de marée, accessible à marée haute avec échouage des navires. Rien ne permet d'affirmer qu'il dispose de structures construites mais l'utilisation du bois et de la pierre envisagée en 1256 pour les ports mineurs de Crémanfleur et Fiquefleur confirment qu'elles peuvent exister. En 1189, il est fait mention du droit de l'abbaye de Grestain, en liaison avec le bénéfice du varech, de porter dans l'église de l'abbaye le corps et l'argent de tout homme mort dans un navire ancré entre l'Épine de Berville et Honfleur, témoignage que certains navires mouillent à l'ancre et sont peut-être déchargés grâce à des bateaux.

46 Homonyme de l'ancêtre des Bertran du XI ${ }^{\mathrm{e}}$ siècle.

47 C. BRÉARD, L'abbaye de Notre-Dame de Grestain, op. cit., PJ VI.

48 Ibid., PJ VII ; il peut s'agir d'un prêt déguisé compte tenu de la possibilité du vendeur de récupérer les masures sous condition de rendre l'argent avant la Saint-Jean-Baptiste 1234.

49 Voir aussi le plan de la collection Gaignières, le plus ancien conservé, rendant compte de la situation avant les travaux du XVII ${ }^{\mathrm{e}}$ siècle. Voir N. PALlu, Honfleur et son arrière-pays, op. cit., vol. 1, p. 34.

50 J. Mallon, Un port normand au Moyen Age..., notes au verso des pages 27-29, p. 35-44. 
Quoi qu'il en soit de la topographie exacte, la juridiction dont disposait Grestain sur la Seine s'étendait jusqu'au voisinage immédiat du port de Honfleur. C'est ce que confirme l'acte de 1287 lorsque l'abbé de Grestain qualifie le fleuve de " nostre eaue de Sainne » entre l'Épine de Berville et Noirport et qu'il est ajouté que l'abbaye ne peut « faire aucune maneuvre parquoy la venue ne le chemin de son [Robert Bertran] port de Honefleu puisse estre empesché ne destorbé en aucune manière ", ce qui s'explique aisément par l'extrême proximité entre Noirport et Honfleur. En outre, cette définition des limites de juridiction en 1287 trouve place dans un acte de composition après un conflit entre les Bertran et l'abbaye à propos de ports de Fiquefleur et Crémanfleur. Il y a de façon évidente des rivalités qui tiennent à la maîtrise des ports ce dont témoignent explicitement les sources.

\section{LES PORTS : ENJEUX DE POUVOIR ET RIVALITÉS ÉCONOMIQUES}

En 1256, dans le premier accord avec l'abbaye de Grestain à propos des ports de Fiquefleur et Crémanfleur, Robert Bertran est qualifié de dominus de Honfleur. À cette date, la famille dispose de nombreux biens dans le pays d'Auge et le Cotentin. En 1275, les biens paternels et maternels sont partagés entre Robert et Guillaume son frère ${ }^{51}$; la «baronnie » de Robert est décrite comme assise à Roncheville ${ }^{52}$, Briquebec ${ }^{53}$ et Honfleur. En 1280 et 1283, deux chartes en français d'un des Robert Bertran portent la mention en ma vile de Honfleur ${ }^{4}$. Dès le $\mathrm{XI}^{\mathrm{e}}$ siècle, les Bertran avaient des possessions à Honfleur puisqu'au milieu de ce siècle, Robert Bertran, premier membre identifié de la famille, et Suzanne, sa femme, donnent l'église Saint-Étienne de Honfleur aux moines de Saint-Ouen de Rouen selon un acte souscrit par Guillaume le Bâtard entre 1051 et $1066^{55}$. Dans une charte non datée et de tradition incertaine, un autre Robert Bertran confirme cette donation, identifiée comme servant à la fondation du prieuré Notre-Dame de Beaumont ${ }^{56}$. À l'église Saint-Étienne de Honfleur s'ajoutent ici celles de Saint-Georges de Fiquefleur et de Saint-Georges de Pennedepie. Enfin, en 1221, un (autre ?) Robert Bertran confirme encore ces donations parmi lesquelles figurent un revenu de quatre livres sur l'église Saint-Étienne de Honfleur (dont le droit

51 G. SAIge, Cartulaire de la seigneurie de Fontenay le Marmion, Monaco, Imprimerie de Monaco, 1895, appendice, $n^{\circ} 3$, p. 177-179; « Acte 2203 », dans SCRIPTA.

52 Commune de Saint-Martin-aux-Chartrains en 1828. Calvados, arr. Lisieux, cant. Pont-l'Évêque.

53 Manche, arr. Cherbourg-Octeville.

54 C. BRÉARD, Cartulaires de Saint-Ymer-en-Auge et Bricquebec, publiés avec notices, RouenParis, Lestringant-Picard, 1908, n 48, p. 234-235; « Acte 2937 » dans SCRIPTA.

55 Recueil des actes des ducs de Normandie de 911 à 1066, op. cit., n 205, p. 393-394 ; « Acte $1649 »$, dans SCRIPTA.

56 J.-F. Pommeraye, Histoire de l'abbaye royale de Saint-Ouen de Rouen, Rouen, Richard Lallemand, 1662, p. 475-476; "Acte 1381 », dans SCRIPTA ; Beaumont-en-Auge (dpt du Calvados ; can. Pont-l'Évêque). 
de présentation reste aux Bertran), l'église Saint-Georges de Fiquefleur, un revenu de 8 livres issu de la prévôté de Honfleur, 40 sous sur la prévôté de Pennedepie et 10 sous sur les cens du même lieu ${ }^{57}$. Ces actes attestent que les Bertran disposent de droits dans ce secteur de la rive gauche de la Seine dès le $\mathrm{XI}^{\mathrm{e}}$ siècle, $\mathrm{y}$ compris à Fiquefleur, qu'une prévôté existe à Honfleur dès ce moment dont les revenus sont, au moins en partie, entre leurs mains, et confirment l'interpénétration des juridictions.

Dans les actes de 1082 et 1189, Fiquefleur et Crémanfleur ne sont pas qualifiés de ports. Ils le sont en 1256 dans l'accord entre Robert Bertran et les moines de Grestain pour régler le conflit qui les oppose à leur propos, conflit qui rebondit et aboutit à une nouvelle transaction en 1287, reprenant en partie les décisions de la première. Robert Bertran avait affirmé disposer d'un droit sur ces ports (in quibus dicebamus nos jus habere) alors que les religieux considéraient qu'ils étaient les leurs en raison du don ancien de Robert, comte de Mortain. Si la Croix du Perier, évoquée plus haut, est effectivement située à Fiquefleur, ce pourrait être un élément d'explication de ces prétentions. Laccord de 1256 prévoit que si les ports ne nécessitent pas de travaux, ils relèveront de l'abbaye, de même que cette dernière bénéficiera des coutumes levées sur les navires et les ports ${ }^{58}$. En revanche, si Robert Bertran supporte le tiers des dépenses d'aménagement, utilisant bois ou pierre, il jouira du tiers des fruits. C'est à la fois le témoignage de la concurrence entre les ports et des difficultés d'accès à ces derniers. En 1287, le litige qui oppose les Bertran et le monastère de Grestain nécessite une nouvelle transaction. La seigneurie sur les ports est de nouveau reconnue à Grestain, mais une nouvelle condition est posée. L'abbaye ne peut effectuer de travaux ${ }^{59}$ et Robert Bertran dispose du droit de détruire les aménagements si cette règle n'est pas respectée. De plus, les moines ne peuvent diminuer le montant des coutumes prélevées, ce qui peut paraître étonnant sauf si Robert Bertran cherche ainsi à éviter que les moines ne rendent leurs ports plus attractifs. Les Bertran apparaissent ici en position de force.

Ils sont également en conflit avec le roi mais les quelques sources qui l'attestent sont d'interprétation malaisée. Un jugement de l'Échiquier de la Saint-Michel 1235 tranche un différend entre les marchands de Honfleur et ceux qui viennent de l'extérieur. Il est statué que ces derniers peuvent faire

57 C. BRÉARD, Cartulaires de Saint-Ymer-en-Auge et Bricquebec..., op. cit., n 19, p. $205-207$; «Acte $2919 »$, dans SCRIPTA.

58 [...] et omnes coustumas tam in navibus quam in aliis dictis portubus adjacentibus et accidentibus similiter dimittimus.

59 «les devantdictz ports ne pourront estre amendez desrre en avant par main d'homme par nous ne par nos successeurs ne par autres ne édiffier en quelle manière que ce soit parquoy il puissent este amendez en aucune manière, fors en l'estat où ilz sont se force de mer ou d'eaue ne les amendent sans manœuvre d'homme, fors tant solement que nous pourrons les pons desdiz ports refaire et cureir le buy de nostre moulin si comme le cours de l'eaue le requiert par la raison du moulin » (C. BRÉARD, L'abbaye de Notre-Dame de Grestain, op. cit., PJ XVIII). 
commerce de poisson à Honfleur, tout autant que les marchands de la ville ${ }^{60}$. Cette décision pourrait être liée à une querelle qui oppose les bourgeois de Honfleur et les bourgeois du roi ${ }^{61}$. En effet, à la session de Pâques précédente, un arrêt rappelle la procédure à suivre mais on comprend mal qui sont les bourgeois du roi ici. Deux autres sources donnent peut-être des clés. Le coutumier de la vicomté de l'eau de Rouen fait mention de la coutume des étals au poisson de Honfleur et Fiquefleur sur lesquels les héritiers de Jean des Vignes doivent quatre livres et cinq sous de rente à la vicomté ${ }^{62}$. Le roi dispose donc de droits à Honfleur et Jean des Vignes et ses successeurs, potentiels bourgeois de Rouen, détiennent peut-être ces étals. En outre, en 1281, le parlement de Paris déboute Robert Bertran de sa plainte à propos du préjudice que pourrait porter le port de Grestain, appartenant au roi, à son port de Honfleur ${ }^{63}$. Charles Bréard propose de considérer qu'il s'agit ici de Noirport car il n'y a aucune autre attestation de port à Grestain. L'hypothèse est intéressante mais elle signifie que le roi serait alors maître d'un espace portuaire au cœur de Honfleur, acquis de l'abbaye de Grestain, et que le port continuerait à porter le nom du monastère. Que ce soit pour Grestain ou Noirport, il n'y a pas d'autre attestation explicite d'activités portuaires. Cela donne la mesure de ce qui échappe mais l'on peut rapprocher cette mention de l'acquisition exactement contemporaine du port d'Harfleur par Philippe le Hardi ${ }^{64}$ témoignant de la volonté royale de prendre nettement pied à l'embouchure du fleuve.

La concurrence pour la maîtrise des ports de la rive gauche est ainsi évidente. Les privilèges accordés par les Bertran à Honfleur peuvent, dans ce contexte, être interprétés comme une volonté de capter les activités et de contrarier la concurrence. Ainsi, les serviteurs et les messagers de l'abbaye

60 Recueil de jugements de l'Échiquier au XIII siècle, L. Delisle (éd.), Mémoires de l'Académie des Inscriptions et Belles Lettres de Caen, t. 24, Paris, 1864, ${ }^{\circ}$ 566, p. 131 ; Etablissements et coutumes, assises et arrêts de l'Échiquier de Normandie au treizième siècle, A. J. MARnIER (éd.), Paris, 1839, p. 168. Dans les versions latines éditées par L. Delisle, il s'agit de la possibilité d'acheter des poissons salés alors que dans la version française donnée par A. J. Marnier, il est question de poissons frais qui peuvent être vendus par ces marchands.

61 Judicatum est quod burgenses de Honnefluctu debent prius negare vel cognoscere injuriam quam faciunt burgensibus domini regis quam habeant diem competentem ad respondendum, et sunt in misericordia quoniam nolebant respondere et pro judicio super hoc expectato (Recueil de jugements de l'Échiquier au XIII siècle, op. cit., n 560, p. 130 ; Établissements et coutumes..., op. cit., p. 167).

62 C.-M. de Robillard de Beaurepaire, De la Vicomté de l'eau de Rouen, op. cit., art. LXXI, p. 358 , et p. 371 ; en revanche, la mention d'une dîme sur la pêche détenue par le monastère de Grimsby à Honfleur est une erreur d'interprétation (N. VINCENT, «The English Monasteries and their French Possessions ", dans Cathedrals, Communities and Conflict in the Anglo-Norman World, Woodbridge Boydell Press, 2011, p. 221-239) : le port de Houflet, cité dans l'acte comme un port d'Henri II dont rien ne permet de penser qu'il a possédé directement Honfleur, est bien plutôt Houflet, Holflete à Stallingborough voisin justement du monastère de Grimsby (voir E. G. Kimball, Some Sessions of the Peace of Lincolnshire, 1381-1396, vol. II, The Lincoln Record Society, Hereford, 1962, p. 23).

63 C. BrÉARD, L'abbaye de Notre-Dame de Grestain..., op. cit., P. J. XVII, p. 220-221.

64 L. DelisLe, Cartulaire normand, op. cit., n 971, p. 247 ; «Acte 3900 », dans SCRIPTA ; L. JEANMARIE, A. Dubois, «Harfleur et Leure, intérêts communs et concurrence (XIII ${ }^{\mathrm{e}}-\mathrm{XIV}^{\mathrm{e}}$ siècles) », colloque $L e$ Havre avant Le Havre, 14-15 septembre 2017, Harfleur, D. SANSY (dir.), Presses universitaires de Rouen et du Havre (à paraître). 
de Montivilliers sont-ils exonérés de coutumes prélevées sur leur passage (in portagio de Honefleto omnes consuetudines sui passagii) dans un acte qui pourrait dater de 1100 mais que Léopold Delisle attribue à la fin du XII ${ }^{\mathrm{e}}$ siècle ${ }^{65}$. En 1283, deux actes distincts exemptent les moines de Beaumont-en-Auge ${ }^{66}$ et ceux de Fécamp ${ }^{67}$ du « travers » de la Seine. Les premiers sont en outre libérés de la coutume sur les achats effectués à Honfleur à l'usage du prieuré, donc en dehors d'éventuelles activités de commerce. Robert Bertran revendiquait justement, avant l'accord de 1287, le droit de travers sur les ports de Fiquefleur et Crémanfleur, ce que l'abbaye de Grestain contestait. Il y a là le témoignage que Honfleur et les petits ports voisins constituent une entrée vers la Normandie occidentale ou, dans l'autre sens, un débouché vers la rive droite de la Seine. La question de la traversée du fleuve est en effet une donnée essentielle et il faut remonter loin vers l'amont, au moins jusqu'à Quillebeuf pour que les deux rives se rapprochent.

En outre, les activités de Honfleur peuvent être mises en évidence par les revenus qui sont tirés de la prévôté, espace de perception des droits seigneuriaux, notamment liés au commerce ${ }^{68}$ dont un tarif copié en 1527 est conservé $^{69}$. On a déjà évoqué les huit livres cédées au XI ${ }^{\mathrm{e}}$ siècle au prieur de Beaumont-en-Auge. En 1228, Robert Bertran, donne 100 sous tournois, à prendre annuellement sur la prévôté de Honfleur, pour fonder l'anniversaire de sa sœur Isabelle, jadis dame de la Roche, dans l'église de la Trinité de la Roche $^{70}$. En 1260, Catherine de Say, femme d'Henri le Maréchal, donne 40 sous à l'abbaye Saint-Étienne de Fontenay à percevoir sur cette même prévôté $^{71}$. Surtout, en 1270, Henri le Maréchal, écuyer et seigneur d'Argentan, vend à Robert Bertran une rente qu'il percevait sur la prévôté en raison des droits de sa mère Catherine. Le prix est de 1400 livres pour un revenu annuel de 148 livres $^{72}$. Henri le Maréchal, seigneur d'Argentan, est un des membres

65 C. BrÉARd, Cartulaires de Saint-Ymer-en-Auge et Bricquebec, op. cit., n I, p. 189-190 ; «Acte 2901 », dans SCRIPTA). Léopold Delisle considère que le millésime n'a peut-être pas été recopié entièrement dans le cartulaire. Niermeyer fait du terme portagium un synonyme de portaticus et donc en l'occurrence une redevance d'amarrage, un droit de port. Du Cange en donne plusieurs définitions, pas toutes liées à un port, et notamment le fait de transporter des marchandises par mer. Le Dictionary of Medieval Latin from British Sources évoque un droit levé sur le transport de biens ou l'utilisation d'un port.

66 C. BRÉARD, Cartulaires de Saint-Ymer-en-Auge et Bricquebec, op. cit., n 52, p. 240-241; « Acte $2941 »$, dans SCRIPTA.

67 E. de Fréville, Mémoire sur le commerce maritime de Rouen, depuis les temps les plus reculés jusqu'à la fin du XVI siècle, Rouen, Le Brument, 1857, t. II, PJ XXVIII.

68 L. JEAn-Marie, «Un espace de prélèvement des coutumes sur le commerce : ville, banlieue et prévôté de Caen », Le Moyen Âge, 1/ 2014, t. CXX, p. 95-122.

69 P. BRÉARD, «Coutume et Prévôté de Honfleur au Moyen Âge », Bulletin de la Société historique de Lisieux, $\mathrm{n}^{\circ}$ 17, 1909, p. 5-20.

70 C. Bréard, Cartulaires de Saint-Ymer-en-Auge et Bricquebec, op. cit., n 20, p. 208, 1228 ; «Acte $2920 »$, dans SCRIPTA.

71 P. Thomas, Histoire de la ville de Honfleur, op. cit., p. 34.

72 C. BréArd, Cartulaires de Saint-Ymer-en-Auge et Bricquebec, op. cit., n 37, p. 227 ; « Acte $3932 »$, dans SCRIPTA. 
de la famille qui a tenu l'office de maréchal auprès du roi pendant plusieurs générations. D'après l'acte, ces revenus lui viennent de sa mère Catherine mais on ne sait comment ils lui sont parvenus. Y aurait-il ici la trace d'une donation royale confirmant l'existence de droits directs du roi à Honfleur ? Par cette transaction, Robert Bertran pourrait chercher à affermir son contrôle sur Honfleur. La somme élevée traduit en outre l'importance des activités commerciales.

À la fin du XIII' ${ }^{\mathrm{e}}$ siècle, des indices laissent donc penser qu'il y a une volonté des Bertran d'imposer Honfleur comme port majeur du secteur. La concomitance des actes suggère une politique volontariste cherchant à éliminer de potentiels concurrents ou bien une réaction face à un développement déjà amorcé des activités des ports voisins.

Quelques attestations concrètes des activités commerciales à Honfleur peuvent être réunies. En 1198, le compte de l'Échiquier fait état à deux reprises, du vin du roi - 50 tonneaux dans l'un des deux cas - qui doit être conduit de Honfleur à Rouen ${ }^{73}$. Le vin arrivé à Honfleur pouvait venir d'Anjou et Poitou mais il est difficile de dire si le port avait alors un rôle particulier en ce domaine. La question est aussi celle des ruptures de charge et de la difficulté à remonter le cours de la Seine qui nécessite plusieurs marées successives. Dans le même compte, à l'alinéa suivant, figurent des dépenses pour préparer quatre "navées » (navatis) de merrains dans la forêt de Bonneville (sur-Touques) et les transporter par mer afin de construire des écuries au château de Caen et effectuer d'autres réparations ${ }^{74}$. Le port de départ pourrait être Honfleur. En 1255, Robert Bertran accorde à Raoul Le Mire, son bourgeois de Honfleur, une franchise de coutumes, à l'exception de celle des cuirs faits avec les peaux de bœufs et vaches et à condition que ce dernier fournisse un navire et le nécessaire pour la traversée lorsque Robert et ses héritiers auront besoin de se rendre en Angleterre. Une fois le navire parvenu dans un port anglais, le reste du ravitaillement restera à Raoul ${ }^{75}$.

Il est pourtant malaisé de situer l'importance de Honfleur comme port de commerce au XIII ${ }^{\mathrm{e}}$ siècle. Si l'on en juge par les mentions de Honfleurais présents en Angleterre d'après les sources anglaises, ils sont loin d'être très nombreux parmi les Normands. En 1225, Henri III ordonne que deux navires de Honfleur et Quillebeuf, chargés de sel, soient libérés ${ }^{76}$. La même année, il

73 Magni Rotuli Scaccari Normanniae sub regibus Angliae, pars secunda, A.-L. LÉCHAUdÉ D’ANISY (éd.), Mémoires de la Société des antiquaires de Normandie, t. XVI, 1852, p. 33 ; Magni rotuli scaccarii Normanniae sub regibus Angliae, T. StAPLEton (éd.), vol. 2, Londres, Sumptibus Soc. antiq. Londiniensi, 1844, p. 371.

74 Ibid.

75 C. BRÉARD, Cartulaires de Saint-Ymer-en-Auge et Bricquebec, op. cit., n 35, p. 225-226 ; « Acte $2930 »$, dans SCRIPTA.

76 Rotuli litterarum clausarum in turri Londinensi asservati, T. D. HARDY (éd.), vol. 2, Londres, Public Records Commission, 1844, p. 62. 
est demandé aux baillis du port de Seaford qu'ils laissent partir à Honfleur le navire de Honfleur chargé de marchandises et appartenant à Henri Burdun ${ }^{77}$. En 1235, le roi s'adresse à Henri de Trubleville, sénéchal de Gascogne, au sujet d'un bateau de Raoul le Sage de Honfleur et de ses compagnons pris à Suling (probablement les îles Scilly au large de la Cornouaille) par le commandant d'une galée dudit Henri et conduit à Guernesey. Il le somme de le leur rendre et de les laisser s'en aller où ils voudront ${ }^{78}$. Les mentions suivantes se situent un siècle plus tard : 1328 et $1335^{79}$. Au total, elles sont bien moins nombreuses que celles de ressortissants d'autres ports normands, tels Dieppe, Harfleur, Rouen, Caen ou même Barfleur. Il est possible que les activités de Honfleur soient peu tournées vers l'Angleterre. Peut-être les liens sont-ils déjà avec les Espagnols qui figurent comme partenaires commerciaux essentiels dans la coutume de la prévôté et auxquels des privilèges commerciaux sont accordés.

Le port de Honfleur des $\mathrm{XI}^{\mathrm{e}}-\mathrm{XIII}^{\mathrm{e}}$ siècles échappe en partie à la connaissance, à la fois du point de vue des activités, de la topographie et de l'exercice des pouvoirs. En dehors même du caractère disparate des sources, il y a ici le témoignage que la topographie portuaire peut être à ce point évolutive, en raison des modifications naturelles et des aménagements humains, qu'il est plus difficile de reconstituer l'occupation du sol que dans d'autres espaces. C'est particulièrement vrai à l'embouchure de la Seine où les atterrissements sont nombreux, ce dont les petits cours d'eau côtiers, lieux d'installation des ports, subissent les effets. Les sources permettent pourtant de prendre la mesure du potentiel économique de la rive gauche qui associe des espaces divers : l'eau, l'estran - dans lequel on peut inclure les ports à marée -, la " terre sèche ». La diversité de la nature des biens et des droits ainsi que leur enchevêtrement géographique rendent compte de la pluralité des activités et de l'intérêt qu'y portent les pouvoirs seigneuriaux. Ce type d'espace est propre aux partages des juridictions mais, sous réserve d'inventaire, le trait est ici particulièrement prononcé. Ce fait, allié à la difficulté de détermination des bornages dans une zone mouvante, est propice aux empiètements et contestations.

On peut penser qu'au $\mathrm{XI}^{\mathrm{e}}$ siècle, un ensemble plus ou moins cohérent a été démembré et distribué par les ducs et leur entourage, ce que traduit en partie la pancarte conservée pour l'abbaye de Grestain. Il est difficile de dire si ces derniers ont conservé à ce moment des éléments d'autorité directe ou si c'est alors que les Bertran ont été également bénéficiaires de donations. Les deux ne

77 Ibid. p. 90.

78 Calendar of the Close Rolls of the reign of Henry III preserved in the Public Record Office, vol. 3, Londres, HSMO, 1908, p. 71.

79 Calendar of the close rolls preserved in the Public Record Office, Edward III, vol. 1, A.D. 13271330, Londres, HSMO, 1896, p. 318 ; Calendar of the close rolls preserved in the Public Record Office, Edward III, vol. 3, A.D. 1333-1337, Londres, HSMO, 1898, p. 484, p. 507. 
sont pas incompatibles et cela expliquerait qu'au XIII siècle, le roi revendique des droits directs, possibles éléments du domaine ducal passé aux Capétiens après 1204. Pourtant au $\mathrm{XII}^{\mathrm{e}}$ siècle, aucune source ne témoigne de tels droits ducaux. Pour ses besoins propres, le duc s'appuie alors principalement sur Barfleur, Caen-Ouistreham, Dieppe et Rouen qui sont en sa main. C'est à la fin du XIII ${ }^{\mathrm{e}}$ siècle, à l'instar de la rive droite, que le roi capétien semble chercher à pousser son avantage alors qu'au même moment les Bertran veulent s'affirmer comme seigneurs de Honfleur et favoriser l'essor du port, quitte à entraver celui des autres.

Quelle est alors l'importance économique de Honfleur à la fin du $\mathrm{XIII}^{\mathrm{e}}$ siècle ? Les informations fournies par les sources sont ténues. Le nombre de navires requis pour les flottes royales pourrait en être une mesure. Dans le compte de l'armée de mer pour l'Aquitaine de 1296, seules 5 nefs viennent de Honfleur, contre 22 de Dieppe et plus de 50 de Leure, Chef-de-Caux et Harfleur réunis ${ }^{80}$. Six navires de Honfleur et un de Fiquefleur participent à la flotte qui est ensuite défaite à la bataille l'Écluse en 1340 alors que 42 sont fournis par Leure, 27 par Dieppe et 9 par Harfleur ${ }^{81}$. Ces comptes ne sont pas le reflet d'une stricte hiérarchie portuaire mais les données peuvent être considérées comme un indice que le port de Honfleur est encore en émergence.

\section{Résumé}

La définition des juridictions dans un espace terrestre et flovio-maritime : Honfleur et ses environs $\left(\mathrm{XI}^{\mathrm{e}}\right.$ - $\mathrm{XIII}^{\mathrm{e}}$ siècles) - La rive gauche de l'embouchure de la Seine est parsemée de petits ports parmi lesquels émerge Honfleur. L'instabilité de la zone en raison de l'action de la mer, la diversité de son potentiel économique et la variété des droits qui s'exercent sur la terre, l'estran et la zone fluviomaritime nécessitent une stricte détermination géographique des juridictions. Les sources témoignent de l'imbrication de ces dernières et des rivalités entre seigneurs des lieux. Au XIII siècle à tout le moins, les Bertran, seigneurs de Honfleur, cherchent à affirmer leur pouvoir et à développer les activités du port ; cela n'est pas sans difficultés, compte tenu sans doute de la concurrence avec d'autres pouvoirs, dont ceux du roi.

Mots-clés : ports, Seine, Moyen Âge, activités portuaires, espaces juridictionnels.

80 Comptes royaux (1285-1314), R. Fawtier et F. Maillard (éd.). Paris, Impr. Nationale - Libr. Klincksieck (Recueil des historiens de la France. Documents financiers, t. III), t. II, Comptes particuliers et comptes spéciaux ou extraordinaires, 1954, 24761, p. 598-601 ; M. HÉrubel évoque le fait, sans citer de référence, que Honfleur arme 5 nefs et 33 galées (Les origines des ports de la Seine-Maritime, Paris, Société d'éditions géographiques maritimes et coloniales, 1930, p. 152 ; repris par M. TRANCHANT, Les ports maritimes de la France atlantique (XI $-X V^{e}$ siècle), t. 1 : Tableau géohistorique, Rennes, Presses universitaires de Rennes, 2018, p. 146).

81 Documents relatifs au clos des galées de Rouen et aux armées de mer du roi de France de 1293 à 1418, A. Chazelas (éd.), Paris, Bibliothèque nationale, 1977-1978, vol. 2, n XXVII ; L. JEAN-Marie, A. Dubois, « Harfleur et Leure... », op. cit. 


\begin{abstract}
The determination of legal jurisdictions in coastal areas: Honfleur and its region (11th - 13th centuries) - Many small ports dot the left bank of the Seine estuary among which Honfleur becamùe dominant. Tidal movements created uncertainty as to the contours of the seacoast and estuary's shoreline, its economic potential and necessitated an exact geography of the extent of local jurisdictions. Local sources bear witness to conflicts over borders and between local lords. During the 13th century, the Bertran, lords of Honfleur, tried to assert their preeminence and develop the port's activities. This was not easy given the rivalry with other jurisdictions, notably that of the Crown.
\end{abstract}

Key Words : ports, Seine, Middle Ages, shipping, jurisdictions. 